\title{
ON GLOBAL SOLUTIONS AND ASYMPTOTIC BEHAVIOR OF PLANAR MAGNETOHYDRODYNAMICS WITH LARGE DATA
}

\author{
BY
}

\author{
YUXI HU
}

Department of Mathematics, China University of Mining and Technology, Beijing 100083, People's Republic of China

\begin{abstract}
In this paper we consider an initial boundary value problem for planar magnetohydrodynamic compressible flows. By assuming that the adiabatic constant $\gamma$ is sufficiently close to 1 , we prove the existence and uniqueness of global strong solutions with large initial data when all the viscosity, heat conductivity, and diffusivity coefficients are constant. Moreover, the asymptotic behavior of solutions is also investigated.
\end{abstract}

1. Introduction. Magnetohydrodynamics (MHD) concerns the motion of conducting fluids in an electromagnetic field. It covers a wide range of physical objects from liquid metals to cosmic plasmas; see [2], [12, [18, [19, 24]. The governing equations of a planar magnetohydrodynamic compressible flow in Lagrange coordinates take the form

$$
\left\{\begin{array}{l}
v_{t}-u_{y}=0, \\
u_{t}+\left(p+\frac{1}{2}|\mathbf{b}|^{2}\right)_{y}=\left(\frac{\lambda u_{y}}{v}\right)_{y}, \\
\mathbf{w}_{t}-\mathbf{b}_{y}=\left(\frac{\mu \mathbf{w}_{y}}{v}\right)_{y}, \\
(v \mathbf{b})_{t}-\mathbf{w}_{y}=\left(\frac{\nu \mathbf{b}_{y}}{v}\right)_{y}, \\
e_{t}+p u_{y}=\left(\frac{\kappa \theta_{y}}{v}\right)_{y}+\frac{\lambda}{v} u_{y}^{2}+\frac{\mu}{v}\left|\mathbf{w}_{y}\right|^{2}+\frac{\nu}{v}\left|\mathbf{b}_{y}\right|^{2},
\end{array}\right.
$$

where $v$ denotes the specific volume, $u \in \mathbb{R}$ the longitudinal velocity, $\mathbf{w} \in \mathbb{R}^{2}$ the transverse velocity, $\mathbf{b} \in \mathbb{R}^{2}$ the transverse magnetic field and $\theta$ the temperature. $p=p(v, \theta)$, $e=e(v, \theta)$ denote the pressure and internal energy, respectively. $\lambda=\lambda(v, \theta)$ and $\mu=\mu(v, \theta)$ are the viscosity coefficients of the flow, $\nu=\nu(v, \theta)$ is the magnetic diffusivity coefficient of the magnetic field, $\kappa=\kappa(v, \theta)$ is the heat conductivity coefficient. The system (1.1) is a three-dimensional magnetohydrodynamic flow which is uniform in the transverse directions; see [3].

There have been a number of studies on MHD by physicists and mathematicians because of its physical importance, complexity, rich phenomena, and mathematical challenges; see [1 [5, 8, 15, 16, 21, 27, 29] and the reference cited therein. In this paper, we

Received April 14, 2014 and, in revised form, August 17, 2014.

2010 Mathematics Subject Classification. Primary 35B40, 35Q35, 76N10.

E-mail address: yxhu86@163.com 
consider a perfect gas for magnetohydrodynamic flow, that is,

$$
p=R \frac{\theta}{v}, \quad e=C_{v} \theta=\frac{R \theta}{\gamma-1}
$$

where $R$ is the gas constant, $C_{v}$ is the heat capacity of the gas at constant volume and $\gamma>1$ is the adiabatic constant.

The system (1.1) is supplemented with the following initial and boundary conditions:

$$
\begin{aligned}
& (v, u, \mathbf{w}, \mathbf{b}, \theta)(y, 0)=\left(v_{0}, u_{0}, \mathbf{w}_{0}, \mathbf{b}_{0}, \theta_{0}\right), \quad \forall y \in \Omega, \\
& \left.\left(u, \mathbf{w}, \mathbf{b}, \theta_{x}\right)\right|_{\partial \Omega}=0,
\end{aligned}
$$

where $\Omega=(0,1)$ and the initial data (1.3) should be compatible with the boundary conditions (1.4).

Now, we recall some well-posedness results for the problem (1.1)-(1.4). The existence and uniqueness of local smooth solutions were proved firstly in [26], while the existence of global smooth solutions with small smooth initial data was shown in [15]. The large-time behavior of solutions was studied in [21], where the exponential stability of solutions was established. The global existence of weak solutions was also studied in 9. Under the technical condition that $\kappa(v, \theta)$ satisfies

$$
C^{-1}\left(1+\theta^{q}\right) \leq \kappa(v, \theta) \equiv \kappa(\theta) \leq C\left(1+\theta^{q}\right)
$$

for some $q \geq 2$, Chen and Wang in [4] obtain the existence, uniqueness and Lipschitz continuous dependence of global strong solutions to (1.1)-(1.4) with large initial data. A similar result is obtained in [3] for real gases. Fan, Jiang and Nakamura [7] obtained global weak solutions to system (1.1)-(1.4) with large initial data and heat conductivity satisfying (1.5) with $q \geq 1$. Very recently, $\mathrm{Hu}$ and $\mathrm{Ju} 11$ extended the above results to the case $q>0$; also see [6] for solutions with vaccum. However, as mentioned in [10], the global existence of classical solution to the full perfect MHD equations with large data remains unsolved when all the viscosity, heat conductivity, and diffusivity coefficients are constant.

In the present paper, we assume that all the coefficients $\lambda, \mu, \nu, \kappa, R$ are positive constants. For simplicity, let $\lambda=\mu=\nu=\kappa=R=1$. We shall show that under the condition that $\gamma-1$ is sufficiently small, the global strong solution exists and neither shock waves nor vacuum and concentration appear in finite time with large initial data. Moreover, the asymptotic behavior of solutions is studied, and the exponential stability is established for large time $t$.

We mention that there exist some results concerning the smallness of $\gamma-1$ in the onedimensional Navier-Stokes equation and the Navier-Stokes-Possion equation; see [23], [14, 20], 25]. Our results can be regarded as an extension to the magnetohydrodynamic flow case. 
Our main results are

Theorem 1.1. Suppose that

- the initial data $\left(v_{0}, u_{0}, \mathbf{w}_{0}, \mathbf{b}_{0}, \theta_{0}\right)$ satisfying

$$
\left\|\left(v_{0}, u_{0}, \mathbf{w}_{0}, \mathbf{b}_{0}, \frac{\theta_{0}-1}{\sqrt{\gamma-1}}\right)\right\|_{H^{1}} \leq E_{0},
$$

where $E_{0}$ is a positive constant independent of $\gamma-1$;

- there exist $\gamma-1$-independent positive constants $\underline{V}, \bar{V}, \underline{\Theta}<1, \bar{\Theta}>1$ such that

$$
\underline{V} \leq v_{0}(y) \leq \bar{V}, \quad \underline{\Theta} \leq \theta_{0}(y) \leq \bar{\Theta} ;
$$

- $\gamma-1$ is sufficiently small.

Then there exists a unique global strong solution $(v, u, \mathbf{w}, \mathbf{b}, \theta)$ on $[0,1] \times[0,+\infty)$ satisfying

$$
\left\|\left(v, u, \mathbf{w}, \mathbf{b}, \frac{\theta-1}{\sqrt{\gamma-1}}\right)\right\|_{H^{1}}+\int_{0}^{+\infty}\left(\left\|\left(u_{y}, \mathbf{w}_{y}, \mathbf{b}_{y}, \theta_{y}\right)\right\|_{H^{1}}^{2}+\left\|\left(v_{y}, u_{t}, \mathbf{w}_{t}, \mathbf{b}_{t}, \theta_{t}\right)\right\|^{2}\right) \mathrm{d} \tau \leq C
$$

and for each $(y, t) \in[0,1] \times[0,+\infty)$,

$$
C^{-1} \leq v(y, t) \leq C, \quad \underline{\Theta} \leq \theta(y, t) \leq \bar{\Theta},
$$

where $C>0$ is constant depending only on the initial data.

Theorem 1.2. Let $(v, u, \mathbf{w}, \mathbf{b}, \theta)$ be the solution obtained in Theorem 1.1. Then

$$
\left\|\left(v-v^{*}, u, \mathbf{w}, \mathbf{b}, \theta-\theta^{*}\right)\right\|_{H^{1}} \rightarrow 0 \quad \text { as } t \rightarrow \infty,
$$

where the constants $v^{*}, \theta^{*}$ are determined by

$$
v^{*}=\int_{0}^{1} v_{0} \mathrm{~d} y, \frac{\theta^{*}}{\gamma-1}=\int_{0}^{1}\left(\frac{\theta_{0}}{\gamma-1}+\frac{1}{2}\left(u_{0}^{2}+\mathbf{w}_{0}^{2}+v_{0} \mathbf{b}_{0}^{2}\right)\right) \mathrm{d} y .
$$

Moreover, there are constants $\alpha, T_{0}, C>0$, independent of $t$, such that

$$
\left\|v-v^{*}\right\|_{H^{1}}+\|u\|_{H^{1}}+\|\mathbf{w}\|_{H^{1}}+\|\mathbf{b}\|_{H^{1}}+\left\|\theta-\theta^{*}\right\|_{H^{1}} \leq C e^{-\alpha t}, \quad t \geq T_{0} .
$$

The existence and uniqueness of local solutions can be obtained by the standard Banach fixed point theorem due to the contraction of the operators defined by the linearized problem (cf. 22]). The global existence of solutions will be proved by the method of extending the local solutions with respect to time based on a priori global estimates.

Now, we present the outline of the proof of the main results. To prove Theorem 1.1. the main idea is to assume the a priori estimate on the absolute temperature $\theta$; see (2.1) below. With given estimate for $\theta$, the estimates for both magnetic field and hydrodynamic velocity (see (2.4) $)$ can be obtained, which is crucial in getting uniform (with respect to time $t$ ) lower and upper bounds on $v$ by using the argument initiated in [17] and [13. With uniform bounds for both $\theta$ and $v$ at hand, we can get the estimates for the higher order derivatives of solution $(v, u, \mathbf{w}, \mathbf{b}, \theta)$ by some delicate energy-type estimates. At the end, in order to extend the solution globally in time, we only need to close the a priori assumption (2.1) where we need the smallness of $\gamma-1$. To prove Theorem 1.2, the idea is to use the established global a priori estimates which ensure that the solution $(v, u, \mathbf{w}, \mathbf{b}, \theta)$ decay to a constant state as time $t$ goes to infinity. The 
exponential decay of solution for large time $t$ can be obtained by using the argument initiated in [21].

2. A priori estimates. In this section, we suppose that the local solution $(v, u, \mathbf{w}, \mathbf{b}, \theta)$ has been extended to time $t=T$. We shall derive the necessary a priori estimates and apply the general continuation argument to get a global solution. First, we assume the following a priori estimates for the temperature $\theta$ :

$$
\frac{1}{2} \underline{\Theta}<\theta \leq 2 \bar{\Theta} .
$$

The smallness of $\gamma-1$ is needed to close this a priori estimate.

In the following, $C$ denotes the generic constants which may depend on initial data but not depend on $T$ and $\gamma-1$, not necessarily the same one at two different spaces.

We first have the following basic energy estimate.

LEMma 2.1. Under the conditions in Theorem 1.1, we have for $0 \leq t \leq T$ that

$$
\begin{aligned}
& \int_{0}^{1} \frac{\theta-1-\ln \theta}{\gamma-1}+(v-\ln v-1)+\frac{1}{2}\left(u^{2}+|\mathbf{w}|^{2}+v|\mathbf{b}|^{2}\right) \mathrm{d} y \\
& +\int_{0}^{t} \int_{0}^{1}\left(\frac{u_{y}^{2}}{v \theta}+\frac{\left|\mathbf{w}_{y}\right|^{2}}{v \theta}+\frac{\left|\mathbf{b}_{y}\right|^{2}}{v \theta}+\frac{\theta_{y}^{2}}{v \theta^{2}}\right) \mathrm{d} y \mathrm{~d} \tau \leq C .
\end{aligned}
$$

Proof. By using system (1.1), we have the equality

$$
\begin{gathered}
\left(\frac{\theta-\ln \theta-1}{\gamma-1}+(v-\ln v-1)+\frac{1}{2}\left(u^{2}+|\mathbf{w}|^{2}+v|\mathbf{b}|^{2}\right)\right)_{t}+(p u-\mathbf{w b})_{y} \\
+\frac{u_{y}^{2}+\left|\mathbf{w}_{y}\right|^{2}+\left|\mathbf{b}_{y}\right|^{2}}{v \theta}+\frac{\theta_{y}^{2}}{v \theta^{2}}=\left(\frac{\theta_{y}}{\theta v}+\frac{u u_{y}+\mathbf{w} \mathbf{w}_{y}+\mathbf{b b}_{y}+\theta_{y}}{v}\right)_{y} .
\end{gathered}
$$

In view of (2.1), we notice that

$$
\theta_{0}-\ln \theta_{0}-1 \leq \frac{1}{\underline{\Theta}}\left(\theta_{0}-1\right)^{2} .
$$

Thus, by initial assumption (1.6), we have

$$
\int_{0}^{1} \frac{\theta_{0}-\ln \theta_{0}-1}{\gamma-1} \mathrm{~d} y \leq \frac{1}{\underline{\Theta}} \int_{0}^{1} \frac{\left(\theta_{0}-1\right)^{2}}{\gamma-1} \mathrm{~d} y \leq C .
$$

Now, integrating (2.3) over $[0,1] \times[0, t]$ and using boundary condition (1.4), we get (2.2) immediately.

The following lemma gives the uniform bounds for both magnetic field and hydrodynamic velocity.

LEMMA 2.2 .

$$
\int_{0}^{t} \max _{y \in[0,1]}\left(u^{2}+\mathbf{w}^{2}+|\mathbf{b}|^{2}\right) \mathrm{d} \tau \leq C .
$$


Proof. By using (1.4), (2.1) and Hölder inequality, we have

$$
\begin{aligned}
u^{2}+\mathbf{w}^{2}+\mathbf{b}^{2} & =\left(\int_{0}^{y} u_{y} \mathrm{~d} y\right)^{2}+\left(\int_{0}^{y} \mathbf{w}_{y} \mathrm{~d} y\right)+\left(\int_{0}^{y} \mathbf{b}_{y} \mathrm{~d} y\right)^{2} \\
& \leq\left(\int_{0}^{1}\left|u_{y}\right| \mathrm{d} y\right)^{2}+\left(\int_{0}^{1}\left|\mathbf{w}_{y}\right| \mathrm{d} y\right)^{2}+\left(\int_{0}^{1}\left|\mathbf{b}_{y}\right| \mathrm{d} y\right)^{2} \\
& \leq\left(\int_{0}^{1}\left(\frac{u_{y}^{2}}{v \theta}+\frac{\mathbf{w}_{y}^{2}}{v \theta}+\frac{\mathbf{b}_{y}^{2}}{v \theta}\right) \mathrm{d} y\right)\left(\int_{0}^{1} v \theta \mathrm{d} y\right) \\
& \leq C \int_{0}^{1}\left(\frac{u_{y}^{2}}{v \theta}+\frac{\mathbf{w}_{y}^{2}}{v \theta}+\frac{\mathbf{b}_{y}^{2}}{v \theta}\right) \mathrm{d} y
\end{aligned}
$$

Thus, in view of the energy estimates (2.2), we get (2.4) immediately.

The next lemma shows the uniform upper and lower bounds of $v$.

LEMMA 2.3 .

$$
C^{-1} \leq v(y, t) \leq C
$$

Proof. Let

$$
\phi(y, t)=\int_{0}^{t}\left(\frac{u_{y}}{v}-p-\frac{1}{2}|\mathbf{b}|^{2}\right)(y, \tau) \mathrm{d} \tau+\int_{0}^{y} u_{0}(x) \mathrm{d} x .
$$

Then

$$
\phi_{t}=\frac{u_{y}}{v}-p-\frac{1}{2}|\mathbf{b}|^{2}, \phi_{y}=u \text {. }
$$

Therefore, using equation (1.1) 1 , we have

$$
(v \phi)_{t}-(u \phi)_{y}=u_{y}-\theta-\frac{1}{2} v \mathbf{b}^{2}-u^{2} .
$$

Integrating the above equation over $[0,1] \times[0, t]$ and using the boundary conditions (1.4), we have

$$
\int_{0}^{1} v \phi \mathrm{d} y=\int_{0}^{1} v_{0} \phi_{0} \mathrm{~d} y-\int_{0}^{t} \int_{0}^{1}\left(\theta+\frac{1}{2} v|\mathbf{b}|^{2}+u^{2}\right) \mathrm{d} y \mathrm{~d} \tau .
$$

On the other hand, by equation (1.1) 1 and the boundary conditions (1.4), we have

$$
\int_{0}^{1} v \mathrm{~d} y=\int_{0}^{1} v_{0} \mathrm{~d} y=v^{*}
$$

By continuity of $\phi$, for any $t>0$, there exists $a(t) \in[0,1]$ such that

$$
\phi(a(t), t)=\frac{1}{v^{*}} \int_{0}^{1} v \phi(y, t) \mathrm{d} y .
$$

By definition of $\phi$ and using equation (1.1) ${ }_{1}$, we have

$$
\begin{aligned}
\phi(a(t), t) & =\int_{0}^{t}\left(\frac{u_{y}}{v}-p-\frac{1}{2}|\mathbf{b}|^{2}\right)(a(t), \tau) \mathrm{d} \tau+\int_{0}^{a(t)} u_{0}(x) \mathrm{d} x \\
& =\ln v(a(t), t)-\ln v_{0}(a(t))-\int_{0}^{t}\left(p+\frac{1}{2}|\mathbf{b}|^{2}\right)(a(t), \tau) \mathrm{d} \tau+\int_{0}^{a(t)} u_{0}(x) \mathrm{d} x .
\end{aligned}
$$


Thus, we get

$$
\begin{aligned}
& \frac{v_{0}(a(t))}{v(a(t), t)} \exp \left\{\int_{0}^{t}\left(p+\frac{1}{2}|\mathbf{b}|^{2}\right)(a(t), \tau) \mathrm{d} \tau\right\} \\
& =\exp \left\{\frac{1}{v^{*}} \int_{0}^{t} \int_{0}^{1}\left(\theta+\frac{1}{2} v|\mathbf{b}|^{2}+u^{2}\right) \mathrm{d} y \mathrm{~d} \tau+\int_{0}^{a(t)} u_{0}(x) \mathrm{d} x-\frac{1}{v^{*}} \int_{0}^{1} v_{0} \phi_{0} \mathrm{~d} y\right\} .
\end{aligned}
$$

Now we derive the explicit formula for $v$ by using a similar argument as in [17]. Integrating (1.1) $)_{2}$ with respect to $t$ and using the equation $(1.1)_{1}$, we have

$$
u(y, t)-u_{0}(y)+\int_{0}^{t}\left(p+\frac{1}{2}|\mathbf{b}|^{2}\right)_{y}(y, \tau) \mathrm{d} \tau=\frac{\mathrm{d}}{\mathrm{d} y}\left(\ln v(y, t)-\ln v_{0}(y)\right) .
$$

Now, doing integration with respect to $y$ at fixed $t$ from the point $a(t)$ to an arbitrary $y \in[0,1]$, we obtain

$$
\begin{aligned}
& \int_{a(t)}^{y}\left(u(x, t)-u_{0}(x)\right) \mathrm{d} x+\int_{0}^{t}\left(p+\frac{1}{2}|\mathbf{b}|^{2}\right)(y, \tau) \mathrm{d} \tau-\int_{0}^{t}\left(p+\frac{1}{2}|\mathbf{b}|^{2}\right)(a(t), \tau) \mathrm{d} \tau \\
& =\ln v(y, t)-\ln v(a(t), t)-\ln v_{0}(y)+\ln v_{0}(a(t)) .
\end{aligned}
$$

Using (2.6), we rewrite the above equation as

$$
\frac{1}{v(y, t)} \exp \left\{\int_{0}^{t} p(y, \tau) \mathrm{d} \tau\right\}=\frac{1}{v_{0}(y)} Y(t) B(y, t) D(y, t)
$$

where

$$
\begin{aligned}
& Y(t)=\exp \left\{\frac{1}{v^{*}} \int_{0}^{t} \int_{0}^{1}\left(\theta+\frac{1}{2} v|\mathbf{b}|^{2}+u^{2}\right) \mathrm{d} y \mathrm{~d} \tau\right\}, \\
& B(y, t)=\exp \left\{\int_{a(t)}^{y}\left[u_{0}(x)-u(t, x)\right] \mathrm{d} x+\int_{0}^{a(t)} u_{0}(x) \mathrm{d} x-\frac{1}{v^{*}} \int_{0}^{1} v_{0} \phi_{0} \mathrm{~d} y\right\}, \\
& D(y, t)=\exp \left\{-\int_{0}^{t} \frac{1}{2}|\mathbf{b}|^{2}(y, \tau) \mathrm{d} \tau\right\} .
\end{aligned}
$$

Multiplying (2.9) by $\theta$, integrating over $[0, t]$, and recalling that $p=\frac{\theta}{v}$, we have that

$$
\exp \left\{\int_{0}^{t} p(y, \tau) \mathrm{d} \tau\right\}=1+\frac{1}{v_{0}(y)} \int_{0}^{t} Y(\tau) B(y, \tau) D(y, \tau) \theta(y, \tau) \mathrm{d} \tau .
$$

Putting (2.10) into (2.9), we finally get that

$$
v(y, t)=v_{0}(y) Y(t)^{-1} B^{-1}(y, t) D^{-1}(y, t)\left(1+\frac{1}{v_{0}} \int_{0}^{t} Y(\tau) B(y, \tau) D(y, \tau) \theta(y, \tau) \mathrm{d} \tau\right) .
$$

From Lemma 2.1 and Lemma 2.2 we easily get that

$$
C^{-1} \leq B(y, t) \leq C, \quad C<D(y, t)<1 .
$$


Thus, by using (2.1), (2.11) and (2.12), we have

$$
\begin{aligned}
v(y, t) & \leq C+C \int_{0}^{t} \exp \left\{-\frac{1}{v^{*}} \int_{\tau}^{t} \int_{0}^{1}\left(\theta+\frac{1}{2} v|\mathbf{b}|^{2}+u^{2}\right) \mathrm{d} y \mathrm{~d} \tau\right\} \\
& \leq C+C \int_{0}^{t} \exp \{-C(t-\tau)\} \mathrm{d} \tau \leq C .
\end{aligned}
$$

On the other hand, by using (1.7), (2.1), (2.11), (2.12), and Lemma 2.1, we have that for any $t \geq 1$,

$$
\begin{aligned}
v(y, t) & \geq C \int_{0}^{t} \exp \left\{-\frac{1}{v^{*}} \int_{\tau}^{t} \int_{0}^{1}\left(\theta+\frac{1}{2} v|\mathbf{b}|^{2}+u^{2}\right) \mathrm{d} y \mathrm{~d} \tau\right\} \\
& \geq C \int_{0}^{t} \exp \{-C(t-\tau)\} \mathrm{d} \tau \geq C(1-\exp \{-C t\}) \geq C
\end{aligned}
$$

and for $t<1$,

$$
v(y, t) \geq C \exp \left\{-\frac{1}{v^{*}} \int_{0}^{t} \int_{0}^{1} \theta+\frac{1}{2} v|\mathbf{b}|^{2}+u^{2} \mathrm{~d} y \mathrm{~d} \tau\right\} \geq C .
$$

Therefore, the proof of this lemma is finished.

Using (2.1), Lemma 2.1 and Lemma 2.3, we have the following estimates.

LEMMA 2.4 .

$$
\int_{0}^{t} \int_{0}^{1}\left(u_{y}^{2}+\left|\mathbf{w}_{y}\right|^{2}+\left|\mathbf{b}_{y}\right|^{2}+\theta_{y}^{2}\right) \mathrm{d} y \mathrm{~d} \tau \leq C .
$$

The following lemma gives the estimates of derivatives of $v$.

LEMMA 2.5 .

$$
\int_{0}^{1} v_{y}^{2} \mathrm{~d} y+\int_{0}^{t} \int_{0}^{1} v_{y}^{2} \mathrm{~d} y \mathrm{~d} \tau \leq C .
$$

Proof. We rewrite the momentum equation $(1.1)_{2}$ as

$$
\left(u-\frac{v_{y}}{v}\right)_{t}=-\left(p+\frac{1}{2}|\mathbf{b}|^{2}\right)_{y} .
$$

Multiplying the above equation by $u-\frac{v_{y}}{v}$ and integrating the result, we have

$$
\begin{aligned}
& \frac{1}{2} \int_{0}^{1}\left(u-\frac{v_{y}}{v}\right)^{2}(y, t) \mathrm{d} y \\
= & \frac{1}{2} \int_{0}^{1}\left(u-\frac{v_{y}}{v}\right)^{2}(y, 0) \mathrm{d} y-\int_{0}^{t} \int_{0}^{1}\left(-\frac{\theta v_{y}}{v^{2}}+\frac{\theta_{y}}{v}+\mathbf{b b}_{y}\right)\left(u-\frac{v_{y}}{v}\right) \mathrm{d} y \mathrm{~d} \tau \\
\leq & C-\int_{0}^{t} \int_{0}^{1} \frac{\theta v_{y}^{2}}{v^{3}} \mathrm{~d} y \mathrm{~d} \tau+\int_{0}^{t} \int_{0}^{1} \frac{\theta v_{y} u}{v^{2}} \mathrm{~d} y \mathrm{~d} \tau-\int_{0}^{t} \int_{0}^{1}\left(\frac{\theta_{y}}{v}+\mathbf{b b}_{y}\right)\left(u-\frac{v_{y}}{v}\right) \mathrm{d} y \mathrm{~d} \tau .
\end{aligned}
$$

Using Young inequality, (2.1), Lemma 2.2 and Lemma 2.3, we have

$$
\begin{aligned}
\int_{0}^{t} \int_{0}^{1} \frac{\theta v_{y} u}{v^{2}} \mathrm{~d} y \mathrm{~d} \tau & \leq \frac{1}{2} \int_{0}^{t} \int_{0}^{1} \frac{\theta v_{y}^{2}}{v^{3}} \mathrm{~d} y \mathrm{~d} \tau+C \int_{0}^{t} \max _{y \in[0,1]} u^{2} \int_{0}^{1} \frac{\theta}{v} \mathrm{~d} y \mathrm{~d} \tau \\
& \leq \frac{1}{2} \int_{0}^{t} \int_{0}^{1} \frac{\theta v_{y}^{2}}{v^{3}} \mathrm{~d} y \mathrm{~d} \tau+C .
\end{aligned}
$$


On the other hand, using (2.1) and Lemmas 2.1] 2.4, we have

$$
\begin{aligned}
& \int_{0}^{t} \int_{0}^{1} \frac{\theta_{y}}{v}\left(u-\frac{v_{y}}{v}\right) \mathrm{d} y \mathrm{~d} \tau \\
& \leq \int_{0}^{t} \int_{0}^{1} \frac{\theta_{y}^{2}}{v \theta^{2}} \mathrm{~d} y \mathrm{~d} \tau+\int_{0}^{t} \max _{y \in[0,1]} u^{2} \int_{0}^{1} \frac{\theta^{2}}{v} \mathrm{~d} y \mathrm{~d} \tau+\frac{1}{4} \int_{0}^{t} \int_{0}^{1} \frac{\theta v_{y}^{2}}{v^{3}} \mathrm{~d} y \mathrm{~d} \tau+C \int_{0}^{t} \int_{0}^{1} \frac{\theta_{y}^{2}}{\theta v} \mathrm{~d} y \mathrm{~d} \tau \\
& \leq C+\frac{1}{4} \int_{0}^{t} \int_{0}^{1} \frac{\theta v_{y}^{2}}{v^{3}} \mathrm{~d} y \mathrm{~d} \tau
\end{aligned}
$$

and

$$
\begin{aligned}
\int_{0}^{t} \int_{0}^{1} \mathbf{b b}_{y}\left(u-\frac{v_{y}}{v}\right) \mathrm{d} y \mathrm{~d} \tau & \leq \int_{0}^{t} \int_{0}^{1}\left|\mathbf{b}_{y}\right|^{2} \mathrm{~d} y \mathrm{~d} \tau+C \int_{0}^{t} \max _{y \in[0,1]}|\mathbf{b}|^{2} \int_{\mathbb{R}}\left(u-\frac{v_{y}}{v}\right)^{2} \mathrm{~d} y \mathrm{~d} \tau \\
& \leq C+C \int_{0}^{t} \max _{y \in[0,1]}|\mathbf{b}|^{2} \int_{\mathbb{R}}\left(u-\frac{v_{y}}{v}\right)^{2} \mathrm{~d} y \mathrm{~d} \tau
\end{aligned}
$$

So, summing up the above estimates, we get

$$
\int_{0}^{1}\left(u-\frac{v_{y}}{v}\right)^{2} \mathrm{~d} y+\int_{0}^{t} \int_{0}^{1} \frac{\theta v_{y}^{2}}{v^{3}} \mathrm{~d} y \mathrm{~d} \tau \leq C+C \int_{0}^{t} \max _{y \in[0,1]}|\mathbf{b}|^{2} \int_{\mathbb{R}}\left(u-\frac{v_{y}}{v}\right)^{2} \mathrm{~d} y \mathrm{~d} \tau .
$$

Using Lemma 2.2 and Gronwall's inequality, we get

$$
\int_{0}^{1}\left(u-\frac{v_{y}}{v}\right)^{2} \mathrm{~d} y+\int_{0}^{t} \int_{0}^{1} \frac{\theta v_{y}^{2}}{v^{3}} \mathrm{~d} y \mathrm{~d} \tau \leq C .
$$

Therefore, in view of (2.1), Lemma 2.1 and Lemma 2.3, the estimate (2.18) follows immediately.

Next, we give the estimates on derivatives of $\mathbf{w}, \mathbf{b}$. For convenience, we present the following interpolation inequalities, which will be frequently used in the coming text, for any $\delta>0$ :

$$
\begin{gathered}
|f|^{2} \leq\left(1+\delta^{-1}\right) \int_{0}^{1}|f|^{2} \mathrm{~d} y+\delta \int_{0}^{1}\left|f_{y}\right|^{2} \mathrm{~d} y \\
\left|f_{y}\right|^{2} \leq\left(1+\delta^{-1}\right) \int_{0}^{1}\left|f_{y}\right|^{2} \mathrm{~d} y+\delta \int_{0}^{1}\left|f_{y y}\right|^{2} \mathrm{~d} y .
\end{gathered}
$$

LEMMA 2.6.

$$
\begin{aligned}
& \int_{0}^{1}\left(\left|\mathbf{w}_{y}\right|^{2}+\left|\mathbf{b}_{y}\right|^{2}\right) \mathrm{d} y \\
& +\int_{0}^{t} \int_{0}^{1}\left(\left|\mathbf{w}_{y y}\right|^{2}+\left|\mathbf{b}_{y y}\right|^{2}+\left|\mathbf{b}_{y}\right|^{4}+\left|\mathbf{w}_{y}\right|^{4}+\left|\mathbf{w}_{t}\right|^{2}+\left|\mathbf{b}_{t}\right|^{2}\right) \mathrm{d} y \mathrm{~d} \tau \leq C, \\
& \max _{(y, \tau) \in[0,1] \times[0, t]}(|\mathbf{w}|+|\mathbf{b}|) \leq C .
\end{aligned}
$$


Proof. Multiplying equation $(1.1)_{3}$ by $\mathbf{w}_{y y}$, integrating the result, using (2.17), (2.18) and (2.23), we have

$$
\begin{aligned}
& \frac{1}{2} \int_{0}^{1} \mathbf{w}_{y}^{2}(y, t) \mathrm{d} y \\
= & \frac{1}{2} \int_{0}^{1} \mathbf{w}_{y}^{2}(y, 0) \mathrm{d} y-\int_{0}^{t} \int_{0}^{1} \frac{\mathbf{w}_{y y}^{2}}{v} \mathrm{~d} y \mathrm{~d} \tau+\int_{0}^{t} \int_{0}^{1} \frac{v_{y} \mathbf{w}_{y} \mathbf{w}_{y y}}{v^{2}} \mathrm{~d} y \mathrm{~d} \tau-\int_{0}^{t} \int_{0}^{1} b_{y} \mathbf{w}_{y y} \mathrm{~d} y \mathrm{~d} \tau \\
\leq & C-\int_{0}^{t} \int_{0}^{1} \frac{\mathbf{w}_{y y}^{2}}{v} \mathrm{~d} y \mathrm{~d} \tau+\int_{0}^{t} \int_{0}^{1}\left(\left|\mathbf{b}_{y}\right|+\left|v_{y}\right|\left|\mathbf{w}_{y}\right|\right) \mathbf{w}_{y y} \mathrm{~d} y \mathrm{~d} \tau \\
\leq & C-\frac{3}{4} \int_{0}^{t} \int_{0}^{1} \frac{\mathbf{w}_{y y}^{2}}{v} \mathrm{~d} y \mathrm{~d} \tau+C \int_{0}^{t} \int_{0}^{1}\left|\mathbf{b}_{y}\right|^{2} \mathrm{~d} y \mathrm{~d} \tau+C \int_{0}^{t} \max _{y \in[0,1]}\left|\mathbf{w}_{y}\right|^{2} \int_{0}^{1} v_{y}^{2} \mathrm{~d} y \mathrm{~d} \tau \\
\leq & C-\frac{1}{2} \int_{0}^{t} \int_{0}^{1} \mathbf{w}_{y y}^{2} \mathrm{~d} y \mathrm{~d} \tau+\int_{0}^{t} \int_{0}^{1}\left|\mathbf{w}_{y}\right|^{2} \mathrm{~d} y \mathrm{~d} \tau \leq C-\frac{1}{2} \int_{0}^{t} \int_{0}^{1} \mathbf{w}_{y y}^{2} \mathrm{~d} y \mathrm{~d} \tau .
\end{aligned}
$$

Thus, we have

$$
\int_{0}^{1}\left|\mathbf{w}_{y}\right|^{2} \mathrm{~d} y+\int_{0}^{t} \int_{0}^{1} \mathbf{w}_{y y}^{2} \mathrm{~d} y \mathrm{~d} \tau \leq C
$$

Rewrite (1.1) 4 as

$$
\mathbf{b}_{t}=-\frac{u_{y}}{v} \mathbf{b}+\frac{1}{v}\left(\mathbf{w}+\frac{\mathbf{b}_{y}}{v}\right)_{y} .
$$

Multiplying the above equation by $\mathbf{b}_{y y}$, integrating the result, using Lemma 2.1, (2.17), (2.22) and (2.23), we have

$$
\begin{aligned}
& \frac{1}{2} \int_{0}^{1}\left|\mathbf{b}_{y}\right|^{2}(y, t) \mathrm{d} y=\frac{1}{2} \int_{0}^{1}\left|\mathbf{b}_{y}\right|^{2}(y, 0) \mathrm{d} y \\
& -\int_{0}^{t} \int_{0}^{1} \frac{\mathbf{b}_{y y}^{2}}{v} \mathrm{~d} y \mathrm{~d} \tau+\int_{0}^{t} \int_{0}^{1} \frac{u_{y} \mathbf{b}-\mathbf{w}_{y}}{v} \mathbf{b}_{y y} \mathrm{~d} y \mathrm{~d} \tau+\int_{0}^{t} \int_{0}^{1} \frac{v_{y} \mathbf{b}_{y} \mathbf{b}_{y y}}{v^{3}} \mathrm{~d} y \mathrm{~d} \tau \\
& \leq C-\frac{3}{4} \int_{0}^{t} \int_{0}^{1} \frac{\mathbf{b}_{y y}^{2}}{v} \mathrm{~d} y \mathrm{~d} \tau \\
& \quad+C \max _{(y, \tau) \in[0,1] \times[0, t]}|\mathbf{b}|^{2} \int_{0}^{t} \int_{0}^{1} u_{y}^{2} \mathrm{~d} y \mathrm{~d} \tau+C \int_{0}^{t} \max _{y \in[0,1]}\left|\mathbf{b}_{y}\right|^{2} \int_{0}^{1} v_{y}^{2} \mathrm{~d} y \mathrm{~d} \tau \\
& \leq C-\frac{1}{2} \int_{0}^{t} \int_{0}^{1} \frac{\mathbf{b}_{y y}^{2}}{v} \mathrm{~d} y \mathrm{~d} \tau+C \int_{0}^{1}|\mathbf{b}|^{2} \mathrm{~d} y+\frac{1}{4} \int_{0}^{1}\left|\mathbf{b}_{y}\right|^{2} \mathrm{~d} y+C \int_{0}^{t} \int_{0}^{1}\left|\mathbf{b}_{y}\right|^{2} \mathrm{~d} y \mathrm{~d} \tau \\
& \leq C-\frac{1}{2} \int_{0}^{t} \int_{0}^{1} \frac{\mathbf{b}_{y y}^{2}}{v} \mathrm{~d} y \mathrm{~d} \tau+\frac{1}{4} \int_{0}^{1}\left|\mathbf{b}_{y}\right|^{2} \mathrm{~d} y .
\end{aligned}
$$

Thus

$$
\int_{0}^{1}\left|\mathbf{b}_{y}\right|^{2} \mathrm{~d} y+\int_{0}^{t} \int_{0}^{1}\left|\mathbf{b}_{y y}\right|^{2} \mathrm{~d} y \mathrm{~d} \tau \leq C
$$


As a consequence of (2.27) and (2.28), we have

$$
\begin{aligned}
\int_{0}^{t} \int_{0}^{1}\left(\left|\mathbf{w}_{y}\right|^{4}+\left|\mathbf{b}_{y}\right|^{4}\right) \mathrm{d} y \mathrm{~d} \tau & \leq \int_{0}^{t}\left(\max _{y \in[0,1]}\left|\mathbf{w}_{y}\right|^{2}+\max _{y \in[0,1]}\left|\mathbf{b}_{y}\right|^{2}\right) \int_{0}^{1}\left(\left|\mathbf{w}_{y}\right|^{2}+\left|\mathbf{b}_{y}\right|^{2}\right) \mathrm{d} y \mathrm{~d} \tau \\
& \leq C \int_{0}^{t} \int_{0}^{1}\left(\left|\mathbf{w}_{y}\right|^{2}+\left|\mathbf{b}_{y}\right|^{2}+\left|\mathbf{w}_{y y}\right|^{2}+\left|\mathbf{b}_{y y}\right|^{2}\right) \mathrm{d} y \mathrm{~d} \tau \leq C
\end{aligned}
$$

Moreover, by equations (1.1) 3 and (2.27), using (2.18), (2.26) and (2.28), we have

$$
\begin{aligned}
\int_{0}^{t} \int_{0}^{1} \mathbf{w}_{t}^{2} \mathrm{~d} y \mathrm{~d} \tau & \leq 2 \int_{0}^{t} \int_{0}^{1} \mathbf{b}_{y}^{2} \mathrm{~d} y \mathrm{~d} \tau+2 \int_{0}^{t} \int_{0}^{1}\left(\frac{\mathbf{w}_{y y}}{v}-\frac{v_{y} \mathbf{w}_{y}}{v^{2}}\right)^{2} \mathrm{~d} y \mathrm{~d} \tau \\
& \leq C+\int_{0}^{t} \max _{y \in[0,1]}\left|\mathbf{w}_{y}\right|^{2} \int_{0}^{1} v_{y}^{2} \mathrm{~d} y \mathrm{~d} \tau \\
& \leq C+C \int_{0}^{t} \int_{0}^{1} \mathbf{w}_{y}^{2} \mathrm{~d} y \mathrm{~d} \tau+C \int_{0}^{t} \int_{0}^{1} \mathbf{w}_{y y}^{2} \mathrm{~d} y \mathrm{~d} \tau \leq C
\end{aligned}
$$

and

$$
\begin{aligned}
\int_{0}^{t} \int_{0}^{1} \mathbf{b}_{t}^{2} \mathrm{~d} y \mathrm{~d} \tau & \leq 2 \int_{0}^{t} \int_{0}^{1} \frac{u_{y}^{2} \mathbf{b}^{2}}{v} \mathrm{~d} y \mathrm{~d} \tau+2 \int_{0}^{t} \int_{0}^{1} \frac{1}{v^{2}}\left(\mathbf{w}+\frac{\mathbf{b}_{y}}{v}\right)_{y}^{2} \mathrm{~d} y \mathrm{~d} \tau \\
& \leq C+C \int_{0}^{t} \max _{y \in[0,1]}\left|\mathbf{b}_{y}^{2}\right| \int_{0}^{1} v_{y}^{2} \mathrm{~d} y \mathrm{~d} \tau \leq C .
\end{aligned}
$$

Thus the proof of this lemma is finished.

Now, multiplying (1.1) $)_{2}$ by $u_{y y}$ and integrating the result, and using (2.1), (2.17), (2.18), (2.24), Lemma 2.1 and Lemma 2.3, we get

$$
\begin{aligned}
& \frac{1}{2} \int_{0}^{1} u_{y}^{2} \mathrm{~d} y+\int_{0}^{t} \int_{0}^{1} \frac{u_{y y}^{2}}{v} \mathrm{~d} y \mathrm{~d} \tau \\
& =\int_{0}^{t} \int_{0}^{1}\left(-\left(p+\frac{1}{2}|\mathbf{b}|^{2}\right)_{y} u_{y y}-\frac{v_{y} u_{y} u_{y y}}{v^{2}}\right) \mathrm{d} y \mathrm{~d} \tau+\frac{1}{2} \int_{0}^{1} u_{y}^{2}(y, 0) \mathrm{d} y \\
& \leq C+\frac{1}{4} \int_{0}^{t} \int_{0}^{1} \frac{u_{y y}^{2}}{v} \mathrm{~d} y \mathrm{~d} \tau+C \int_{0}^{t} \int_{0}^{1} \frac{\theta_{y}^{2}}{v \theta^{2}} \mathrm{~d} y \mathrm{~d} \tau+\int_{0}^{t} \int_{0}^{1} \frac{v_{y}^{2} \theta^{2}}{v^{3}} \mathrm{~d} y \mathrm{~d} \tau+\int_{0}^{t} \max _{y \in[0,1]}\left|u_{y}\right|^{2} \mathrm{~d} \tau \\
& \leq \frac{1}{2} \int_{0}^{t} \int_{0}^{1} \frac{u_{y y}^{2}}{v} \mathrm{~d} y \mathrm{~d} \tau+C .
\end{aligned}
$$

Thus we get

$$
\int_{0}^{1} u_{y}^{2} \mathrm{~d} y+\int_{0}^{t} \int_{0}^{1} u_{y y}^{2} \mathrm{~d} y \mathrm{~d} \tau \leq C
$$

and

$$
\int_{0}^{t} \int_{0}^{1} u_{y}^{4} \mathrm{~d} y \mathrm{~d} \tau \leq \int_{0}^{t} \max _{y \in[0,1]}\left|u_{y}\right|^{2} \int_{0}^{1} u_{y}^{2} \mathrm{~d} y \mathrm{~d} \tau \leq \int_{0}^{t} \int_{0}^{1} u_{y y}^{2} \mathrm{~d} y \mathrm{~d} \tau \leq C .
$$

Using the Sobolev imbedding theorem, we have

$$
\max _{(y, \tau) \in[0,1] \times[0, t]} u \leq C .
$$


Moreover, using equations (1.1) 2 and (2.1), Lemma 2.3. Lemma 2.5, and Lemma 2.6, we have

$$
\int_{0}^{t} \int_{0}^{1} u_{t}^{2} \mathrm{~d} y \mathrm{~d} \tau \leq C
$$

Combining (2.29), (2.30), (2.31) and (2.32) together, we obtain the following lemma.

LEMMA 2.7.

$$
\max _{(y, \tau) \in[0,1] \times[0, t]} u+\int_{0}^{1} u_{y}^{2} \mathrm{~d} y+\int_{0}^{t} \int_{0}^{1}\left(u_{y}^{4}+u_{y y}^{2}+u_{t}^{2}\right) \mathrm{d} y \mathrm{~d} \tau \leq C .
$$

The estimates for the derivatives of $\theta$ are given in the following lemma.

LEMMA 2.8 .

$$
\int_{0}^{1} \frac{\theta_{y}^{2}}{\gamma-1}+\int_{0}^{t} \int_{0}^{1}\left(\theta_{y y}^{2}+\frac{\theta_{t}^{2}}{(\gamma-1)^{2}}\right) \mathrm{d} y \mathrm{~d} \tau \leq C .
$$

Proof. Taking derivatives with respect to $y$ in temperature equation (1.1) ${ }_{5}$, we have

$$
\frac{\theta_{y t}}{\gamma-1}+\left(p u_{y}\right)_{y}=\left(\frac{\theta_{y}}{v}\right)_{y y}+\left(\frac{u_{y}^{2}+\left|\mathbf{w}_{y}\right|^{2}+\left|\mathbf{b}_{y}\right|^{2}}{v}\right)_{y} .
$$

Multiplying the above equation by $\theta_{y}$, integrating the results, using (2.1), (2.18), (2.24), (2.33) and Lemma 2.3, we have

$$
\begin{aligned}
& \int_{0}^{1} \frac{\theta_{y}^{2}}{\gamma-1} \mathrm{~d} y=\int_{0}^{1} \frac{\theta_{0 y}^{2}}{\gamma-1} \mathrm{~d} y-\int_{0}^{t} \int_{0}^{1}\left(\left(\frac{\theta_{y}}{v}\right)_{y}+\left(p u_{y}\right)-\frac{u_{y}^{2}+\left|\mathbf{w}_{y}\right|^{2}+\left|\mathbf{b}_{y}\right|^{2}}{v}\right) \theta_{y y} \mathrm{~d} y \mathrm{~d} \tau \\
& \leq C-\frac{1}{2} \int_{0}^{t} \int_{0}^{1} \frac{\theta_{y y}^{2}}{v} \mathrm{~d} y \mathrm{~d} \tau+\int_{0}^{t} \int_{0}^{1} \theta_{y}^{2} v_{y}^{2} \mathrm{~d} y \mathrm{~d} \tau+\int_{0}^{t} \int_{0}^{1}\left(u_{y}^{2}+u_{y}^{4}+\left|\mathbf{w}_{y}\right|^{4}+\left|\mathbf{b}_{y}\right|^{4}\right) \mathrm{d} y \mathrm{~d} \tau \\
& \leq C-\frac{1}{2} \int_{0}^{t} \int_{0}^{1} \frac{\theta_{y y}^{2}}{v} \mathrm{~d} y \mathrm{~d} \tau+\int_{0}^{t} \max _{y \in[0,1]} \theta_{y}^{2} \mathrm{~d} \tau \\
& \leq C-\frac{1}{4} \int_{0}^{t} \int_{0}^{1} \frac{\theta_{y y}^{2}}{v} \mathrm{~d} y \mathrm{~d} \tau .
\end{aligned}
$$

Thus, we get

$$
\int_{0}^{1} \frac{\theta_{y}^{2}}{\gamma-1} \mathrm{~d} y+\int_{0}^{t} \int_{0}^{1} \frac{\theta_{y y}^{2}}{v} \mathrm{~d} y \mathrm{~d} \tau \leq C .
$$

By using equations (1.1) 5 and (2.1), (2.36), Lemma 2.3, Lemma 2.5, Lemma 2.6 and Lemma 2.7, we get

$$
\int_{0}^{t} \int_{0}^{1} \frac{\theta_{t}^{2}}{(\gamma-1)^{2}} \leq C
$$

So, combining (2.36) and (2.37), we get (2.34), and the proof of this lemma is finished.

3. Proof of Theorem 1.1 and Theorem 1.2. In view of the a priori estimates established in section 2, we only need to close the a priori estimate (2.1) to complete the proof of Theorem 1.1. We need the smallness of $\gamma-1$ in order to do this. Firstly, simple calculations imply the following inequality:

$$
\frac{1}{4 \bar{\Theta}}(\theta-1)^{2} \leq \theta-\ln \theta-1 \text {. }
$$


The above inequality together with the basic energy estimate (2.2) yields

$$
\|\theta-1\|_{L^{2}} \leq C(\gamma-1)^{\frac{1}{2}}
$$

So, by the Sobolev imbedding theorem, we have

$$
\|\theta-1\|_{L^{\infty}} \leq \int_{0}^{1}|\theta-1| \mathrm{d} y+\int_{0}^{1}\left|\theta_{y}\right| \mathrm{d} y \leq\|\theta-1\|_{L^{2}}+\left\|\theta_{y}\right\|_{L^{2}} \leq C(\gamma-1)^{\frac{1}{2}} .
$$

Therefore, choose $\gamma-1$ sufficiently small such that

$$
1+C(\gamma-1)^{\frac{1}{2}} \leq \bar{\Theta}, \quad 1-C(\gamma-1)^{\frac{1}{2}} \geq \underline{\Theta} .
$$

Combining (3.3) and (3.4), we get

$$
\underline{\Theta} \leq \theta(y, t) \leq \bar{\Theta} \text {. }
$$

By the continuity argument, we see that the estimate (2.1) will always hold for $0 \leq t \leq T$, and the proof of Theorem 1.1 is completed.

Now, we show the convergence of $(v, u, \mathbf{w}, \mathbf{b}, \theta)$ to the constant state $\left(v^{*}, 0,0,0, \theta^{*}\right)$ in $H^{1}(0,1)$ as $t$ goes to infinity. To this end, we observe that by (1.1) ${ }_{1}$, Lemmas 2.5]2.8, and the equality

$$
\int_{0}^{1}\left(u_{x} u_{x t}+\mathbf{w}_{x} \mathbf{w}_{x t}+\mathbf{b}_{x} \mathbf{b}_{x t}+\theta_{x} \theta_{x t}\right) \mathrm{d} y=-\int_{0}^{1}\left(u_{x x} u_{t}+\mathbf{w}_{x x} \mathbf{w}_{t}+\mathbf{b}_{x x} \mathbf{b}_{t}+\theta_{x x} \theta_{t}\right) \mathrm{d} y,
$$

we have

$$
\int_{0}^{\infty}\left\{\frac{\mathrm{d}}{\mathrm{d} t}\left(\left\|v_{x}\right\|^{2}+\left\|u_{x}\right\|^{2}+\left\|\mathbf{w}_{x}\right\|^{2}+\left\|\mathbf{b}_{x}\right\|^{2}+\left\|\theta_{x}\right\|^{2}\right)\right\} \mathrm{d} t \leq C,
$$

which combined with Lemmas $2.4[2.5$ yields

$$
\left\|v_{x}\right\|^{2}+\left\|u_{x}\right\|^{2}+\left\|\mathbf{w}_{x}\right\|^{2}+\left\|\mathbf{b}_{x}\right\|^{2}+\left\|\theta_{x}\right\|^{2} \rightarrow 0, \quad \text { as } t \rightarrow \infty .
$$

Using Poincaré's inequality, we have

$$
\left\|v-v^{*}\right\|_{H^{1}}+\|u\|_{H^{1}}+\|\mathbf{w}\|_{H^{1}}+\|\mathbf{b}\|_{H^{1}} \rightarrow 0, \quad \text { as } t \rightarrow \infty .
$$

So, in view of (3.8), in order to complete the proof of the convergence of solutions, it remains to show that

$$
\left\|\theta-\theta^{*}\right\| \rightarrow 0, \quad \text { as } t \rightarrow \infty .
$$

By using equations $(1.1)_{1}-(1.1)_{4}$, we can rewrite equation $(1.1)_{5}$ as

$$
E_{t}+\left[\left(p+\frac{1}{2} \mathbf{b}^{2}\right) u-\mathbf{w b}\right]_{y}=\left(\frac{u u_{y}+\mathbf{w} \mathbf{w}_{y}+\mathbf{b} \mathbf{b}_{y}+\theta \theta_{y}}{v}\right)_{y},
$$

where $E(t)=\frac{\theta}{\gamma-1}+\frac{1}{2}\left(u^{2}+\mathbf{w}^{2}+v \mathbf{b}^{2}\right)$. Recalling the definition of $\theta^{*}$, integrating the above equation over $[0,1] \times[0, t]$, we have

$$
\int_{0}^{1}\left(\frac{\theta}{\gamma-1}+\frac{1}{2}\left(u^{2}+\mathbf{w}^{2}+v \mathbf{b}^{2}\right)\right) \mathrm{d} y=\frac{\theta^{*}}{\gamma-1}
$$


which together with Poincaré's inequality, Lemma 2.3, Lemma 2.6, Lemma 2.7] and (3.7), gives

$$
\begin{aligned}
& \left\|\frac{\theta}{\gamma-1}-\frac{\theta^{*}}{\gamma-1}+\frac{1}{2}\left(u^{2}+\mathbf{w}^{2}+v \mathbf{b}^{2}\right)\right\|^{2} \\
& \leq C\left\|\theta_{x}+u u_{x}+\mathbf{w w}_{x}+v \mathbf{b}_{x}+\frac{1}{2} \mathbf{b}^{2} v_{x}\right\|^{2} \\
& \leq C\left\|\theta_{x}\right\|^{2}+\max _{(y, t) \in[0,1] \times[0, \infty)}\left(u^{2}+\mathbf{w}^{2}+v^{2} \mathbf{b}^{2}+\mathbf{b}^{4}\right)\left(\left\|u_{x}\right\|^{2}+\left\|\mathbf{w}_{x}\right\|^{2}+\left\|\mathbf{b}_{x}\right\|^{2}+\left\|v_{x}\right\|^{2}\right) \\
& \leq C\left(\left\|v_{x}\right\|^{2}+\left\|u_{x}\right\|^{2}+\left\|\mathbf{w}_{x}\right\|^{2}+\left\|\mathbf{b}_{x}\right\|^{2}+\left\|\theta_{x}\right\|^{2}\right) .
\end{aligned}
$$

It follows by the triangle inequality that

$$
\begin{aligned}
\left\|\frac{\theta-\theta^{*}}{\gamma-1}\right\|^{2} & \leq\left\|\frac{\theta}{\gamma-1}-\frac{\theta^{*}}{\gamma-1}+\frac{1}{2}\left(u^{2}+\mathbf{w}^{2}+v \mathbf{b}^{2}\right)\right\|^{2}+\left\|\frac{1}{2}\left(u^{2}+\mathbf{w}^{2}+v \mathbf{b}^{2}\right)\right\|^{2} \\
& \leq C\left(\left\|v_{x}\right\|^{2}+\left\|u_{x}\right\|^{2}+\left\|\mathbf{w}_{x}\right\|^{2}+\left\|\mathbf{b}_{x}\right\|^{2}+\left\|\theta_{x}\right\|^{2}\right) \rightarrow 0, \quad \text { as } t \rightarrow \infty .
\end{aligned}
$$

We know that $v-v^{*}, u, \mathbf{w}, \mathbf{b}, \theta-\theta^{*}$ become small in $H^{1}$-norm for large $t$; thus we can apply an argument similar to those used in 21] to obtain (1.12) in Theorem 1.2 (the exponential convergence of $(v, u, \mathbf{w}, \mathbf{b}, \theta)$ to the constant state as $t$ goes to infinity). Thus the proof of Theorem 1.2 is completed.

\section{REFERENCES}

[1] R. Balescu, Transport processes in plasmas I: Classical transport theory, North-Holland, 1988.

[2] H. Cabannes, Theoretical magnetofluiddynamics, Academic Press, New York, 1970.

[3] Gui-Qiang Chen and Dehua Wang, Global solutions of nonlinear magnetohydrodynamics with large initial data, J. Differential Equations 182 (2002), no. 2, 344-376, DOI 10.1006/jdeq.2001.4111. MR.1900327 (2003d:35212)

[4] Gui-Qiang Chen and Dehua Wang, Existence and continuous dependence of large solutions for the magnetohydrodynamic equations, Z. Angew. Math. Phys. 54 (2003), no. 4, 608-632, DOI 10.1007/s00033-003-1017-z. MR1994028 (2005b:35227)

[5] P. C. Clemmow and J. P. Dougherty, Electrodynamics of particles and plasmas, Addison-Wesley, New York, 1990.

[6] J. S. Fan, S. X. Huang, and F. C. Li, Global strong solutions to the planar compressible magnetohydrodynamic equations with large initial data and vaccum, preprint (2013).

[7] Jishan Fan, Song Jiang, and Gen Nakamura, Vanishing shear viscosity limit in the magnetohydrodynamic equations, Comm. Math. Phys. 270 (2007), no. 3, 691-708, DOI 10.1007/s00220-006-0167-1. MR2276461(2008g:76135)

[8] H. Freistühler and P. Szmolyan, Existence and bifurcation of viscous profiles for all intermediate magnetohydrodynamic shock waves, SIAM J. Math. Anal. 26 (1995), no. 1, 112-128, DOI 10.1137/S0036141093247366. MR1311884 (95j:35183)

[9] David Hoff and Eugene Tsyganov, Uniqueness and continuous dependence of weak solutions in compressible magnetohydrodynamics, Z. Angew. Math. Phys. 56 (2005), no. 5, 791-804, DOI 10.1007/s00033-005-4057-8. MR2184906 (2006k:35230)

[10] Xianpeng $\mathrm{Hu}$ and Dehua Wang, Global existence and large-time behavior of solutions to the threedimensional equations of compressible magnetohydrodynamic flows, Arch. Ration. Mech. Anal. 197 (2010), no. 1, 203-238, DOI 10.1007/s00205-010-0295-9. MR2646819 (2011d:35079)

[11] Yuxi $\mathrm{Hu}$ and Qiangchang Ju, Global large solutions of magnetohydrodynamics with temperaturedependent heat conductivity, Z. Angew. Math. Phys. 66 (2015), no. 3, 865-889, DOI 10.1007/s00033014-0446-1. MR 3347415

[12] A. Jeffrey and T. Taniuti, Non-linear wave propagation. With applications to physics and magnetohydrodynamics, Academic Press, New York-London, 1964. MR0167137 (29 \#4410) 
[13] Song Jiang, On the asymptotic behavior of the motion of a viscous, heat-conducting, onedimensional real gas, Math. Z. 216 (1994), no. 2, 317-336, DOI 10.1007/BF02572324. MR1278427 (95e:35120)

[14] Shuichi Kawashima and Takaaki Nishida, Global solutions to the initial value problem for the equations of one-dimensional motion of viscous polytropic gases, J. Math. Kyoto Univ. 21 (1981), no. 4, 825-837. MR637519 (84d:76042)

[15] Shuichi Kawashima and Mari Okada, Smooth global solutions for the one-dimensional equations in magnetohydrodynamics, Proc. Japan Acad. Ser. A Math. Sci. 58 (1982), no. 9, 384-387. MR694940 (85c:35078)

[16] Shuichi Kawashima and Yasushi Shizuta, Magnetohydrodynamic approximation of the complete equations for an electromagnetic fluid, Tsukuba J. Math. 10 (1986), no. 1, 131-149. MR846424 $(87 \mathrm{~m}: 76070 \mathrm{a})$

[17] A. V. Kazhikhov and V. V. Shelukhin, Unique global solution with respect to time of initial-boundary value problems for one-dimensional equations of a viscous gas (Russian), Prikl. Mat. Meh. 41 (1977), no. 2, 282-291; English transl., J. Appl. Math. Mech. 41 (1977), no. 2, 273-282. MR0468593 (57 \#8425)

[18] A. G. Kulikovskiy and G. A. Lyubimov, Magnetohydrodynamics, Addison-Wesley, Reading, Massachusetts, 1965.

[19] L. D. Laudau and E. M. Lifshitz, Electrodynamics of continuous media, 2nd ed., Pergamon, New York, 1984.

[20] Hongxia Liu, Tong Yang, Huijiang Zhao, and Qingyang Zou, One-dimensional compressible NavierStokes equations with temperature dependent transport coefficients and large data, SIAM J. Math. Anal. 46 (2014), no. 3, 2185-2228, DOI 10.1137/130920617. MR3225502

[21] Tai-Ping Liu and Yanni Zeng, Large time behavior of solutions for general quasilinear hyperbolicparabolic systems of conservation laws, Mem. Amer. Math. Soc. 125 (1997), no. 599, viii+120, DOI 10.1090/memo/0599. MR1357824 (97g:35107)

[22] John Nash, Le problème de Cauchy pour les équations différentielles d'un fluide général (French), Bull. Soc. Math. France 90 (1962), 487-497. MR0149094 (26 \#6590)

[23] Mari Okada and Shuichi Kawashima, On the equations of one-dimensional motion of compressible viscous fluids, J. Math. Kyoto Univ. 23 (1983), no. 1, 55-71. MR692729 (85c:76050)

[24] R. V. Polovin and V. P. Demutskii, Fundamentals of magnetohydrodynamics, Consultants Bureau, New York, 1990.

[25] Zhong Tan, Tong Yang, Huijiang Zhao, and Qingyang Zou, Global solutions to the one-dimensional compressible Navier-Stokes-Poisson equations with large data, SIAM J. Math. Anal. 45 (2013), no. 2, 547-571, DOI 10.1137/120876174. MR3032988

[26] A. I. Vol'pert and S. I. Hudjaev, The Cauchy problem for composite systems of nonlinear differential equations (Russian), Mat. Sb. (N.S.) 87(129) (1972), 504-528. MR0390528 (52 \#11353)

[27] Dehua Wang, Large solutions to the initial-boundary value problem for planar magnetohydrodynamics, SIAM J. Appl. Math. 63 (2003), no. 4, 1424-1441 (electronic), DOI 10.1137/S0036139902409284. MR 1989910 (2004f:76121)

[28] L. C. Woods, Principles of magnetoplasma dynamics, Oxford Science Publications, The Clarendon Press, Oxford University Press, New York, 1987. MR.957923 (89i:76099)

[29] C. C. Wu, Formation, structure, and stability of MHD intermediate shocks, J. Geophys. Res. 95 (1990), 8149-8175. 